\title{
An animal model for Charcot-Marie-Tooth disease type 4B1
}

\author{
Sonja Bonneick ${ }^{1}$, Matthias Boentert ${ }^{2}$, Philipp Berger ${ }^{1}$, Suzana Atanasoski ${ }^{1}$, Ned Mantei ${ }^{1}$, \\ Carsten Wessig ${ }^{3}$, Klaus V. Toyka ${ }^{3}$, Peter Young ${ }^{2}$ and Ueli Suter ${ }^{1, *}$ \\ ${ }^{1}$ Institute of Cell Biology, Department of Biology, Swiss Federal Institute of Technology, ETH-Hönggerberg, \\ $\mathrm{CH}-8093$ Zurich, Switzerland, ${ }^{2}$ Department of Neurology, University of Münster, Münster, Germany and \\ ${ }^{3}$ Department of Neurology, Julius Maximilians-Universität Würzburg, Würzburg, Germany
}

Received August 23, 2005; Revised and Accepted October 14, 2005

\begin{abstract}
Charcot-Marie-Tooth disease (CMT) comprises a family of clinically and genetically very heterogeneous hereditary peripheral neuropathies and is one of the most common inherited neurological disorders. We have generated a mouse model for CMT type 4B1 using embryonic stem cell technology. To this end, we introduced a stop codon into the Mtmr2 locus within exon 9, at the position encoding amino acid 276 of the MTMR2 protein (E276X). Concomitantly, we have deleted the chromosomal region immediately downstream of the stop codon up to within exon 13. The resulting allele closely mimics the mutation found in a Saudi Arabian CMT4B1 patient. Animals homozygous for the mutation showed various degrees of complex myelin infoldings and outfoldings exclusively in peripheral nerves, in agreement with CMT4B1 genetics and pathology. Mainly, paranodal regions of the myelin sheath were affected, with a high degree of quantitative and qualitative variability between individuals. This pathology was progressive with age, and axonal damage was occasionally observed. Distal nerve regions were more affected than proximal parts, in line with the distribution in CMT. However, we found no significant electrophysiological changes, even in aged (16-month-old) mice, suggesting that myelin infoldings and outfoldings per se are not invariably associated with detectable electrophysiological abnormalities. Our animal model provides a basis for future detailed molecular and cellular studies on the underlying disease mechanisms in CMT4B1. Such an analysis will reveal how the disease develops, in particular, the enigmatic myelin infoldings and outfoldings as well as axonal damage, and provide mechanistic insights that may aid in the development of potential therapeutic approaches.
\end{abstract}

\section{INTRODUCTION}

Charcot and Marie in France and Tooth in England described the clinical features of hereditary motor and sensory neuropathies in the late 19th century (reviewed in 1). Thus, this group of disorders is referred to as Charcot-Marie-Tooth (CMT) disease. With an estimated prevalence of 1:2500, CMT is one of the major genetic diseases in clinical neuroscience. Main features are slowly progressive distal and proximal muscle weakness and atrophy. Using clinical, electrophysiological and histological criteria, CMT has been divided into demyelinating (CMT1, CMT3, CMT4) and axonal forms (CMT2).

Reduced nerve conduction velocity (NCV) and conduction failure due to primary segmental demyelination is characteristic of demyelinating neuropathies. The myelin deficit is followed by axonal degeneration leading to muscular atrophy (2,3). Axonal forms of CMT are associated with nearly normal NCV, but loss of myelinated fibers causes a conduction failure in degenerated nerve fibers, resulting in a reduction of the compound action potential amplitude. In this case, the primary insult is thought to affect the axon or neuron, eventually followed by secondary demyelination $(4,5)$.

Initially, it was assumed that defects in Schwann cellspecific proteins would result in demyelinating neuropathies, whereas defects in neuron-specific proteins lead to axonal forms of CMT. However, recent progress in identifying the responsible disease genes and unraveling the complex

\footnotetext{
*To whom correspondence should be addressed. Tel: +41 446333432; Fax: +41 446331190; Email: usuter@cell.biol.ethz.ch
} 
interplay of Schwann cells and axons in peripheral nerves has shown that primary and secondary effects can often not be resolved with certainty (2). Both Schwann cells and neurons may express a particular disease-causing gene, making simple distinctions as to disease contributions by the different cell types difficult.

The severe autosomal recessive CMT4B1 is caused by mutations in myotubularin related protein-2 (MTMR2) (6-8) including missense, nonsense, frameshift and exon-skipping deletions. Disease onset is usually in early childhood, and $\mathrm{NCV}$ is markedly reduced. Histological analysis of nerve biopsies revealed characteristic focally folded myelin sheaths and demyelination, although this pathologic feature is not unique to CMT4B1 $(9,10)$. Mtmr2 is expressed by both neurons and Schwann cells, and their individual cellular contributions to the phenotype are not yet clear $(11,12)$. Recent experimental data obtained by Schwann cell-specific disruption of Mtmr2 indicate a major role of this cell type (13).

MTMR2 is a member of the myotubularin family of dual specific phosphatases. Myotubularin is the founding member of the family, and mutations in the myotubularin gene cause X-linked myotubular myopathy, one of the severe congenital muscle diseases causing a 'floppy infant syndrome' (14). On the basis of sequence and structural analysis, all myotubularins contain characteristic domains: a PH (pleckstrin-homology)/GRAM (glycosyltransferase, Rab-like-GTPase activator and myotubularins) domain, a phosphatase domain and a coiled-coil domain. In MTMR2, a coiled-coil domain mediates dimerization, and, together with the PH/GRAM domain which binds to phosphatidylinositol 3,5-bisphosphate $[\mathrm{PI}(3,5) \mathrm{P} 2]$ and $\mathrm{PI}(5) \mathrm{P}$, is required for membrane targeting (15). MTMR2 also carries a C-terminal PDZ (PSD-95/Dlg/ZO-1)-binding motif that binds to disc-large 1/synapse-associated protein 97 (DLG1)/ (SAP97) (13). An interaction with neurofilament light chain (NF-L) has also been reported (16).

Depending on the functionality of the phosphatase domain, the myotubularins are divided into two subgroups: Those family members with an intact phosphatase motif (Cys-X5Arg) form the group of active phosphatases, whereas some myotubularins have modifications in the active site turning them into inactive phosphatases. Intriguingly, mutations affecting the inactive phosphatase MTMR13/SBF2 are associated with CMT4B2, a neuropathy that is clinically and pathologically indistinguishable from CMT4B1 $(17,18)$. Active and inactive myotubularins tend to physically interact with each other (19-21) and recent evidence suggests that MTMR2 and MTMR13/SBF2 act as a similar functional pair in myelinated peripheral nerves (22) (Berger et al., manuscript in preparation).

Active myotubularins dephosphorylate both $\mathrm{PI}(3,5) \mathrm{P} 2$ and PI(3)P $(11,23,24)$ by removing the phosphate group on the D3 position. MTMR2 displays a higher activity toward $\mathrm{PI}(3,5) \mathrm{P} 2$ (11). The physiological significance of this observation is unclear, although it may be connected to the membrane targeting of MTMR2 mediated by the coiled-coil and PH/GRAM domains. MTMR2 mutations associated with CMT4B1 lead to a loss or strong reduction of phosphatase activity on both substrates, consistent with a crucial role in the disease (11).
Recent reports based on appropriate animal models have provided encouraging prospects for new strategies to ameliorate CMT (25-27). These promising developments underline the value of animal models for our further understanding of basic disease mechanisms, thereby contributing to the evaluation of potential treatments (28). In this report, we describe the generation and analysis of a new animal model of CMT4B1 as a prerequisite for such approaches.

\section{RESULTS}

\section{Disruption of Mtmr2}

We designed a targeting vector for recombination in ES cells to mimic the genetic situation in a CMT4B1 patient at the MTMR2 locus (6). To achieve this goal, a stop codon was introduced into exon 9 at the codon for amino acid 276, which is within the phosphatase domain. In addition, the region downstream of this mutation to within exon 13 was replaced by an IRES-lacZ-tk-neo cassette (Fig. 1A). Southern blot analysis of correctly targeted ES cells and genomic tail DNA, derived from the resulting mutant mice, revealed the expected diagnostic bands (Fig. 1B). Single integration was further confirmed by a lacZ probe (Fig. 1B). Mutant mice obtained from two independent ES cell clones were viable and born with Mendelian frequency. An Mtmr2-derived transcript was readily detected in mutant mice, and, as expected, immunohistochemical analysis on nerve sections suggested that a truncated MTMR2 protein is still expressed by peripheral nerves of homozygous mutant mice (data not shown). Unfortunately, reliable biochemical detection and analysis of this putative protein was not successful with the available reagents.

Mice homozygous for the mutant allele appeared outwardly normal by visual inspection up to the age of 16 months, the oldest stage analyzed. Occasionally, homozygous mutants show an unusual but only mild clamping of the hind limbs after prolonged suspension by the tail. No significant differences were found in gait and performance in the Rotarod test or in grip strength (Fig. 1C). Heterozygous mice were indistinguishable from their wild-type (wt) littermates at all ages.

\section{Altered myelin in homozygous Mtmr2 mutant mice}

On the basis of the pathology described in peripheral nerves of CMT4B1 patients, we started our analysis with this tissue. At the age of 2 months, the morphology of mutant peripheral nerves appeared grossly normal (Fig. 2A-C). However, at higher magnification, we found abnormal Schwann cellaxon units with redundant myelin loops arranged around myelinated, usually large caliber axons, exclusively in homozygous mutants (arrows and arrowhead in Fig. 2F). Myelinated small caliber axons and non-myelinated axons appeared unaffected.

\section{Progression of myelin abnormalities in Mtmr2 mutant mice}

As demyelinating neuropathies follow a progressive time course, we analyzed the focally redundant myelin at the age 
A
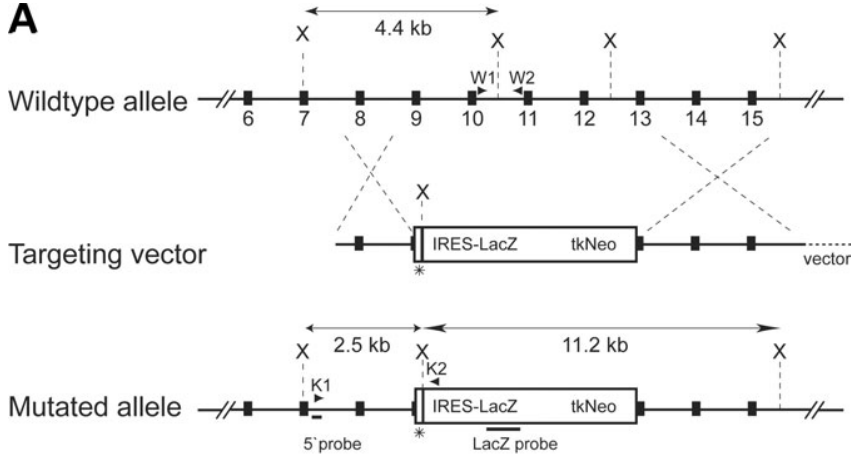

B

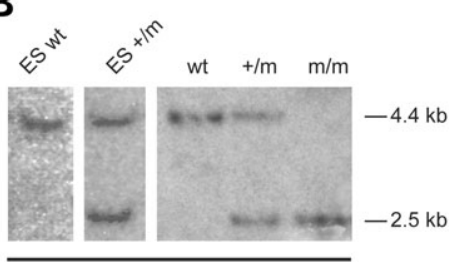

5' probe

C

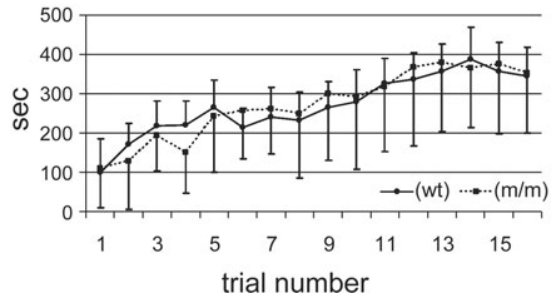

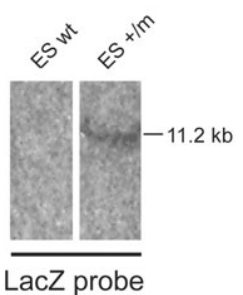

D

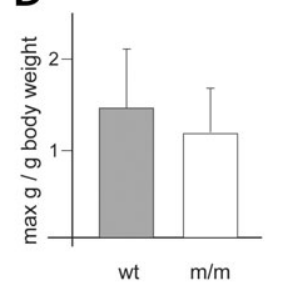

Figure 1. Targeted disruption of Mtmr2. (A) Schematic depiction of the Mtmr 2 wt allele, targeting construct and mutated allele. Solid boxes represent exons 6-15 of Mtmr2. After homologous recombination, a stop codon, indicated by an asterisk, is inserted into exon 9 at the same position as described for a CMT4B1 patient. The resulting premature translation termination leads to a putative protein of 275 amino acids. Additionally, the IRES-lacZ-tk-neo cassette replaces the genomic region from exon 9 to within exon 13, encoding major parts of the PTP and SID motifs. Positions of $\mathrm{XbaI}(\mathrm{X})$ restriction sites used for Southern blot analysis are indicated. Bars indicate the 5'-external probe and the lacZ probe. PCR primer pairs (arrowheads) W1 plus W2 (wt PCR) and K1 plus K2 (mutant PCR) were used for genotyping. Note that introns and exons are not drawn to scale. (B) Southern blot analysis of wt and recombined ES cells, and wt, heterozygous mutant $(+/ \mathrm{m})$ and homozygous mutant $(\mathrm{m} / \mathrm{m})$ mice. Genomic DNA isolated from ES cells or tail biopsies was digested with $X b a \mathrm{I}$ and hybridized with a radioactive probe. Because of the insertion of an additional $X b a \mathrm{I}$ restriction site with the lacZ-tk-neo cassette (see A), the mutant allele $(\mathrm{m})$ gives a shorter $2.5 \mathrm{~kb}$ fragment compared with the wt $4.4 \mathrm{~kb}$ fragment when probed with the $5^{\prime}$-external probe. The lacZ probe was used to confirm single integration. (C) A Rotarod test was used to assess the motor activity of 9-month-old wt, Mtmr $2+/ m$ and $M t m r 2 \mathrm{~m} / \mathrm{m}$ mice. Mice were tested four times per day every $2 \mathrm{~h}$ on four consecutive days. The time spent on the rotating rod versus the trial number was plotted. Averages for eight mice per genotype are shown. No significant differences were observed. (D) The same mice as for (C) were used for grip strength analysis. The $y$-axis shows the maximum weight the mice could hold in relation to the body weight. There is no significant difference in the forelimb grip strength of wt and Mtmr $2 \mathrm{~m} / \mathrm{m}$ mice.

of 3 weeks and at 2, 4 and 6 months. At 3 weeks, some rare affected myelin sheaths were already detectable in association with large myelinated fibers in homozygous mutants (Fig. 3E). At later stages, the number and complexity of affected
Schwann cell-axon units were increased, and small myelinated fibers were also affected (Fig. 3E-H). We did not observe obvious signs of demyelination and remyelination, such as thin myelin sheaths associated with large axons or onion bulb formation.

To quantify redundant myelin formation and its progression with age, we determined the fraction of abnormal Schwann cell-axon units in the pectineus nerve of wt and homozygous mutant animals at 3 weeks and 6 months of age. Older mutants tended to have more fibers affected with redundant myelin loops than did younger mice. We also noted, however, a high variability in the degree of pathology among individual mutant mice at both ages (Fig. 4A). Furthermore, we compared the axonal caliber in relation to myelin thickness in the sciatic nerve of homozygous mutants versus wt mice, but found no significant alterations (Fig. 4B).

\section{Distal parts of peripheral nerves of $M t m r 2$ mutants are more severely affected than proximal areas}

Hereditary demyelinating neuropathies often more strongly affect distal rather than proximal segments of peripheral nerves (29). Although it has been speculated that this could be due to impaired transport along the axon from the neuronal cell body to the periphery (2), this hypothesis remains to be confirmed. To examine whether distal nerves are more affected than proximal segments also in our model, we analyzed peripheral nerves of 6-month-old homozygous mutants at the root level and in distal nerve branches. Indeed, we found more pronounced redundant myelin formation in distal motor (gluteus) and sensory (saphenous) nerves compared with the nerve roots (Fig. 5, compare A with $\mathrm{C}$ and $\mathrm{B}$ with D). Motor and sensory branches did not differ significantly in the severity of the myelin phenotype.

In CMT4B1 patients, the phenotype is restricted to the PNS with no obvious defects in the CNS. In agreement, we found no alterations in the optic nerve or in the spinal cord of mutant mice (data not shown).

\section{Structure of redundant myelin in Mtmr2 mutants is highly complex}

We performed ultrastructural analyses to further characterize the focally folded redundant myelin loops that are specific hallmarks of Mtmr 2 mutants and highly reminiscent of the pathological features found in CMT4B1 (7). Figure 6A presents a collection of the different forms of abundant myelin figures found in mutant nerves. In the most common formation, one or several myelin loops were arranged around a core-myelinated large caliber fiber (a). These myelin loops contained axoplasma-like structures (Fig. 7) (data not shown). Abnormal myelin occurred not only as loops but also as finger-like elongations wrapped around the axon (b). However, this appearance may also be due to a myelin loop indented into the original axon. Comma-like extensions of the core-myelinated axon were often observed, although they are not specific to the mutant (c). In mutant nerves, these extensions were often large in size and long enough to surround aberrant myelin loops of the same Schwann cell-axon unit (d). Redundant myelin loops were also found 

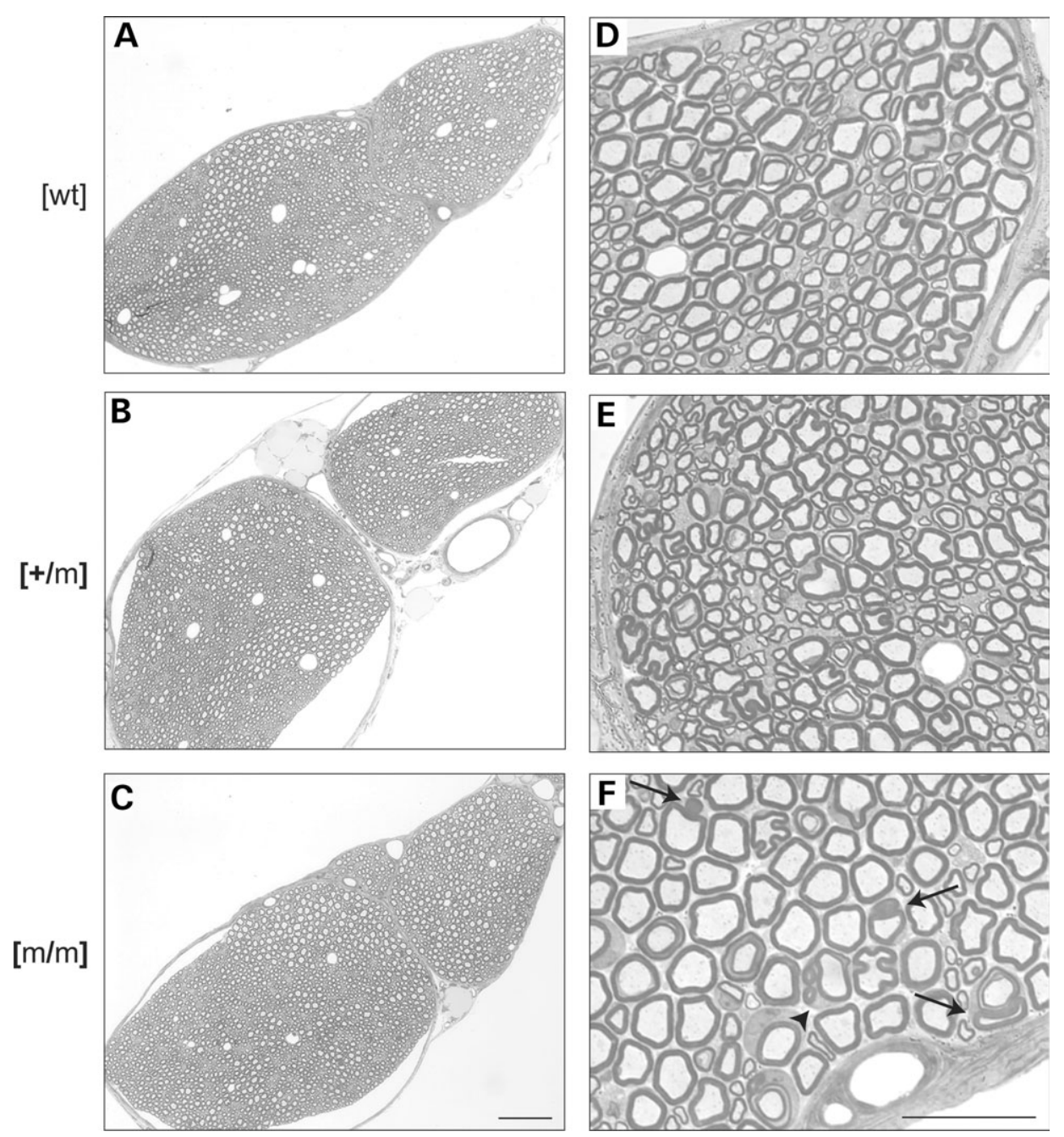

Figure 2. Histological analysis of sciatic nerves. Sciatic nerve cross-sections of wt (A and $\mathbf{D}), M t m r 2+/ \mathrm{m}(\mathbf{B}$ and $\mathbf{E})$ and $M t m r 2 \mathrm{~m} / \mathrm{m}(\mathbf{C}$ and $\mathbf{F}) \mathrm{mice}$ at $2 \mathrm{months}$ of age. The gross overall morphology of heterozygous and homozygous mutant nerves appears unaltered compared with that of wt littermates (B and C versus A). At higher magnification, redundant myelin is detectable specifically around axons of Mtmr $2 \mathrm{~m} / \mathrm{m}$ mice (arrowhead and arrows in F). Myelinated fibers of $M t m r 2+/ m$ or wt mice are not affected (compare F with D and E). Note that the additional myelin loops and the large caliber axon in (F) marked with an arrowhead are surrounded by continuous cytoplasm, indicating that they originate from the same Schwann cell. Scale bars for (A-C): $100 \mu m$; for (D-F): $25 \mu \mathrm{m}$.

inside the axon (e) or inside another aberrant myelin loop (f). Single loops were present but also multiple loops nested into each other (e and f). Degenerating Schwann cell-axon units (g) were seen only very rarely.

To gain further insights into the structure of the redundant myelin, we analyzed teased fibers and longitudinal sections of sciatic nerves. In osmium tetroxide-fixed teased fibers, a conspicuous myelin thickening around the nodes of Ranvier was apparent (Fig. 6Ba and b). In longitudinal sections, we found that a substantial number of nodes exhibited abnormal myelin. Redundant myelin was folded inside the original axon (c and e) or in an outwardly directed fashion (g). Infoldings and outfoldings occurred focally and were not mutually exclusive. They did not usually affect the nodes symmetrically. Focally folded myelin arranged in an outward direction contained not only myelin but also outcroppings of axons, both continuous with the original axon-myelin structure ( $f$ and $g$ ). This is consistent with the observation that the number of lamellae appeared to be the same in outwardly folded myelin as in the core-myelinated fiber (Fig. 6Ah).

\section{Immunohistochemical analysis of Mtmr2 mutant nerves}

To confirm that outfoldings in Mtmr2 mutant nerves indeed contain axonal structures, we analyzed affected nodal regions by immunohistochemistry. S100 delineates the cytoplasm of Schwann cells, and NF-L labels axons. The S100 staining allowed us to define formations consisting of a myelinated large caliber axon and a redundant myelin loop (Fig. 7Aa, c, h and i). Both structures are surrounded by the S100-positive structure, indicating one Schwann cell. The NF-L staining marks not only the large caliber axon but also the inside of the aberrant myelin loop (Fig. 7Af and i). This indicates that the outwardly directed focal myelin foldings are elongations of core myelin and that axonal structures are contained within both of them. 

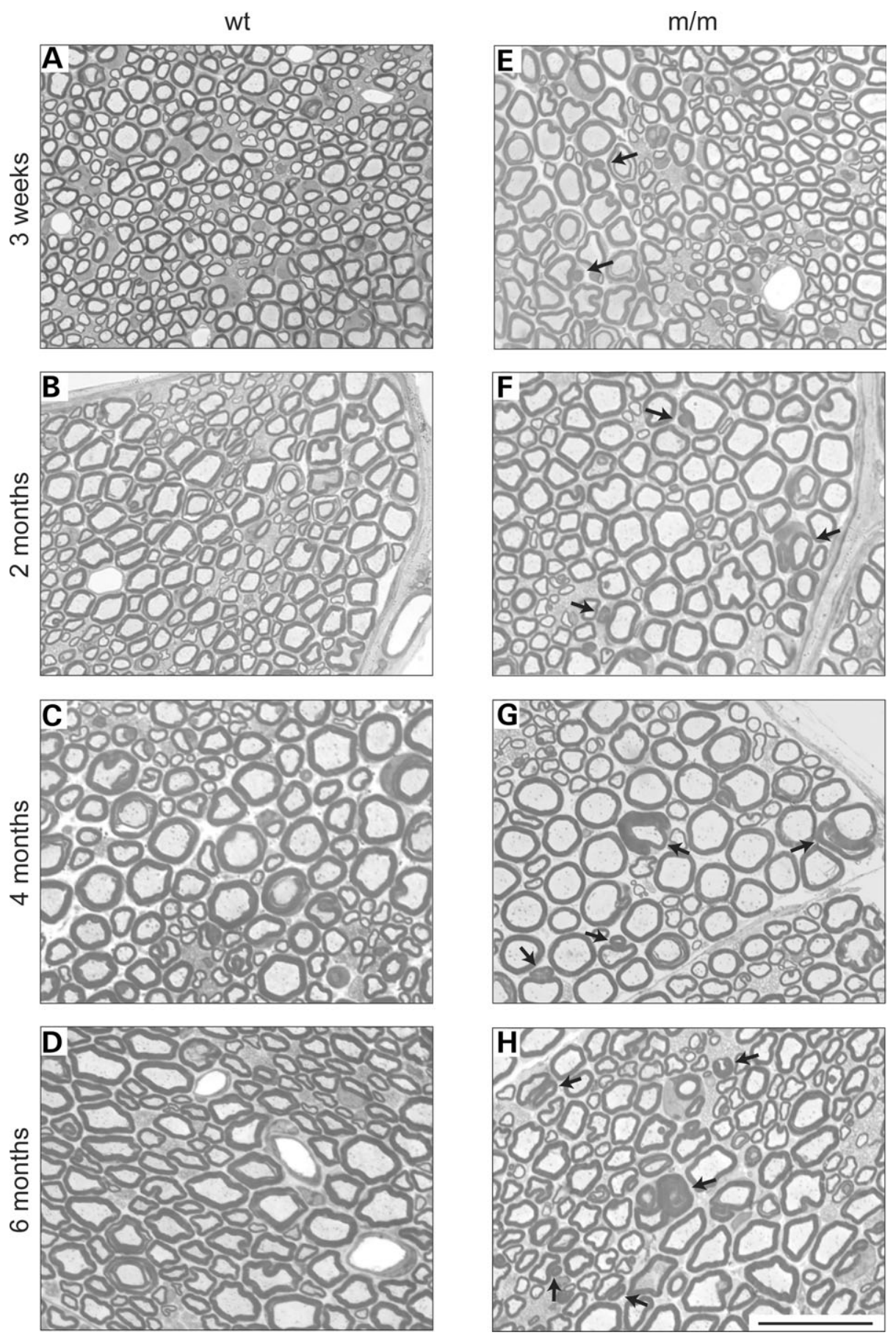

Figure 3. Time course analysis of sciatic nerves. Sciatic nerve cross-sections of wt (A-D) and $M t m r 2 \mathrm{~m} / \mathrm{m}(\mathbf{E}-\mathbf{H})$ mice. The morphology was compared at the age of 3 weeks (A and E), 2 months (B and F), 4 months (C and G) and 6 months (D and H). Three animals were analyzed per age and genotype, and representative pictures are shown. Occasional large caliber axons affected with redundant myelin loops were detected in Mtmr2 $\mathrm{m} / \mathrm{m}$ mice at 3 weeks of age (E). Progressively more affected axons were found in older mutant mice, with both the size and the number of redundant myelin loops increasing. Scale bar for (A-H): $25 \mu \mathrm{m}$.

Redundant myelin was prominent in nodal regions, and we therefore analyzed mutant nodes of Ranvier in more detail. Immunohistochemical analysis of Caspr, a marker for paranodal axolemma, the juxtaparanodal marker Kv1.2 and myelin staining with myelin basic protein (MBP) was used (Fig. 7B). On teased fiber preparations, we observed nodes of Ranvier with normal distribution of these markers (Fig. 7Ba-d). Abnormal nodes of Ranvier were discernible by a particularly pronounced MBP staining ( $\mathrm{g}$ and $\mathrm{k}$ ). The paranodal localization of Caspr was not altered by the redundant myelin (e and i). Similarly, Kv1.2 was usually still restricted to its juxtaparanodal localization (f). Occasionally, 


\section{A}
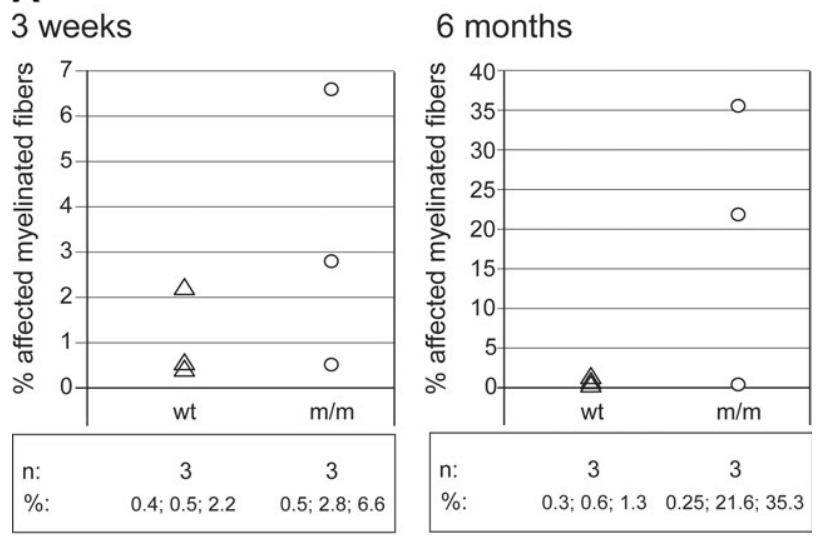

\section{B}

\section{3 weeks}

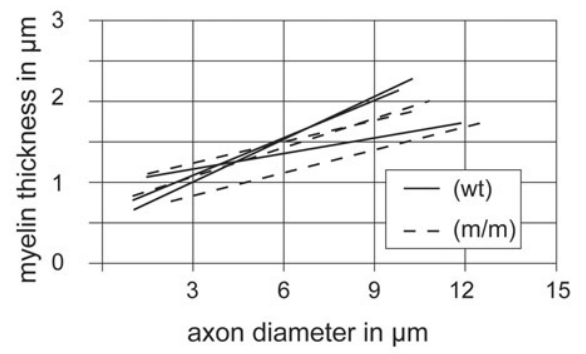

6 months

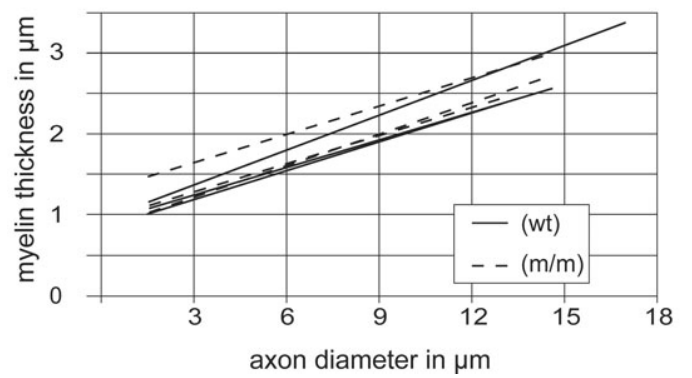

Figure 4. Quantification of myelinated fibers and redundant myelin loops. (A) Quantification of fibers exhibiting redundant myelin loops at 3 weeks and 6 months of age. For each stage, the pectineus nerve was isolated from three mice per genotype. Ten semithin sections per nerve were used for quantification. The mean value per mouse is shown as triangles for wt and as circles for Mtmr2 $\mathrm{m} / \mathrm{m}$ mice. The number of affected myelinated fibers tends to increase in Mtmr2 $\mathrm{m} / \mathrm{m}$ mice during aging (note different scales for the two graphs), although there is great variation among different mice. (B) Relation between axon diameter and myelin thickness of 3-week- and 6-month-old mice. For each age, the sciatic nerves of three animals per genotype were analyzed. Myelin thickness was determined and plotted as a function of the corresponding axon diameter. Each line represents the trend line for one mouse. Slope and intercept did not differ significantly between wt and $M t m r 2 \mathrm{~m} / \mathrm{m}$ mice at either age.

however, the Kv1.2-positive juxtaparanodal domain appeared to be extended into areas of MBP-positive myelin ( $\mathrm{j}$ and $\mathrm{l}$ ).

\section{Electrophysiology of Mtmr2 mutant mice}

We examined peripheral nerves of Mtmr2 mutants by electrophysiological methods to assess the impact of the morphological abnormalities on nerve function. Mice were first examined at 8 months of age and again at 16 months. In contrast to humans with CMT4B1, we found no significant differences between mutant and wt animals with regard to compound muscle action potential (CMAP) amplitudes (after both proximal and distal stimulation), nerve conduction velocities or F-wave latencies (Fig. 8).

\section{DISCUSSION}

We describe a novel animal model for CMT4B1. These animals display prominent and complex myelin infoldings and outfoldings, the pathological hallmark of this particular form of CMT. This pathology appears to be progressive with time, although we observed a large variability between individual mutant mice. Surprisingly, signs of demyelination like thinly myelinated large caliber axons or onion bulb formation were not detected, and axonal degeneration was only occasionally found. These structural alterations were not reflected in disordered nerve conduction in our mutants up to the age of 16 months.

It is informative to compare our mouse mutants with the genotype and phenotype of the human disease CMT4B1 and with the recently described Mtmr2 null mutant mice (13). We will first consider the myelin pathology followed by the electrophysiological findings.

The described myelin pathologies are largely comparable in all three cases and thus, the Mtmr 2 mutant described here and Mtmr2 null mice will be very useful to follow up on the development and cellular basis of this peculiar feature. The myelin alterations might be related to the dephosphorylation of PI(3)P and/or PI(3,5)P2 by MTMR2, which has been demonstrated in vitro $(11,30)$ and is likely to regulate $\mathrm{PI}(3) \mathrm{P}$ or $\mathrm{PI}(3,5) \mathrm{P} 2$ levels also in vivo. The specificity for the endosomal substrates $\mathrm{PI}(3) \mathrm{P}$ and $\mathrm{PI}(3,5) \mathrm{P} 2$ suggests that MTMR2 may regulate endosomal trafficking including endocytosis, vacuolar transport and membrane trafficking $(31-34)$. CMT4B1 mutations in MTMR2, including the E276Stop mutant introduced into the mice described in this report, lead either to a complete loss of the phosphatase activity towards $\mathrm{PI}(3) \mathrm{P}$ and $\mathrm{PI}(3,5) \mathrm{P} 2$ or to a severe reduction of the enzymatic activity (11). How this relates to the formation of aberrant myelin foldings in MTMR2 mutants remains to be determined. An intriguing hypothesis has been put forward based on the association of MTMR2 with Dlg1/SAP97 (13). This scaffolding protein plays a role in membrane trafficking (35), and disruption of the Dlg1/MTMR2 complex could lead to excess membrane transport to the affected paranodes and thereby cause redundant myelin formation. Alternatively or concomitantly, the loss of phosphatase function of the MTMR2/Dlg1, or of a conceivable MTMR2/MTMR13/Dlg1 complex, may play a critical role in the process of myelin alteration.

Reduced NCV is a hallmark of the demyelinating neuropathies, including CMT4B1 (2). Six-month-old Mtmr2 null mice also showed reduced NCV and prolonged F-wave latencies (13). Interestingly, the mutant mice reported here do not exhibit significant electrophysiological alterations up to the age of 16 months. This discrepancy might be related to differences in how the mutant mice were generated (null mutant versus introduction of a stop codon at position 276). 

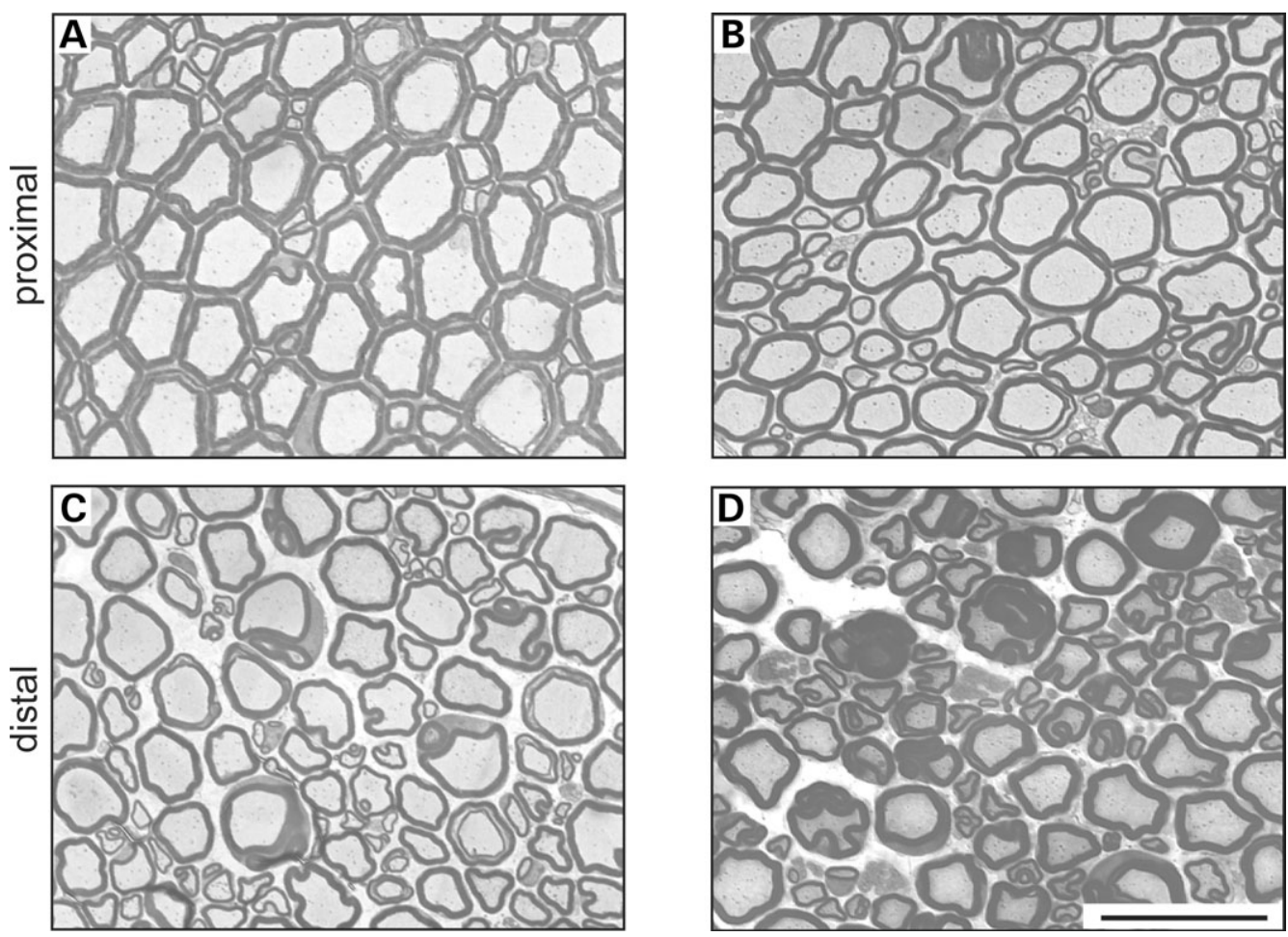

Figure 5. Histological analysis of distal and proximal PNS nerves. Cross-sections of proximal (A: ventral roots, B: dorsal roots) and distal (C: gluteus nerve, D: saphenous nerve) nerve segments of Mtmr $2 \mathrm{~m} / \mathrm{m}$ mice at 6 months of age. Myelin infoldings and outfoldings were rarely found in proximal areas, whereas they occur often in distal nerves. Scale bar for (A-D): $25 \mu \mathrm{m}$

Alternatively, mouse strain differences may be responsible for these seemingly contradictory observations.

Several other observations are also subtly different in Mtmr 2 null mice versus the mutants described here. First, Mtmr 2 null mice have not been obtained with a Mendelian distribution (13), whereas this was the case for our mutants. Secondly, a testis phenotype has been described in Mtmr2 null mice and is consistent with an earlier described azoospermia in an isolated CMT4B1 patient (14) and with the testis phenotype of Mtmr5 null mice (36) [MTMR5 is a binding partner of MTMR2 (21)]. In our case, this pathology was highly variable between individual males (data not shown). On the basis of these findings, together with the observed variability of the nerve pathology, we favor the hypothesis that strong modifier genes are involved in the modulation of the phenotype, varying either within the mixed background of the individual mutant lines or between the two disease models. These two mouse models may thus offer an opportunity to analyze the potential role of such modifier genes in a well-defined disease. To approach this important question systematically, it will be necessary to produce congenic strains, followed by a detailed comparative analysis.

Both the Mtmr 2 null mice and our mutants are less severely affected than might have been expected from the symptomology of CMT4B1. It is a general observation that in mouse models of demyelinating forms of CMT, the mutants are less affected than are humans suffering from the corresponding clinical condition (3). Although the Mtmr2 null mice exhibited electrophysiological abnormalities, against the background of the human disease they were rather mild. Moreover, the reduction of F-wave conduction was disproportionately minor when compared with the reported NCV of more distal nerve segments. The marked reduction of NCV observed in CMT4B patients may be related to different mutations or to the role of important modifier genes in humans. Demyelination and secondary axonal loss might also be more pronounced because of the much longer nerves in humans, with impaired axonal transport potentially playing an important role (2). The phenotype may also increase in severity with age, and the lifetime of a mouse may not be long enough to allow full development of the disease. Although we have observed a progressive development of myelin abnormalities, both qualitatively and quantitatively, we have not found indications for a neurological worsening in the mutants up to 16 months of age. Functional redundancy and compensation by other myotubularins may be involved, although quantitative RT-PCR analysis of mutant nerves of young adult animals has not revealed consistent alterations in the level of either the closely related family members MTM and MTMR1 or the MTMR2-binding partners MTMR5 and MTMR13 (data not shown).

In conclusion, we have generated a new animal model for CMT4B1, mimicking a mutation found in human patients with typical CMT4B1. The effects on myelin structure were similar to those seen in humans, although the clinical impact was not so severe. There were obvious differences in comparison to the previously described null mutant mouse with regard to electrophysiological parameters, frequency of homozygous mutants among offspring and variability of the phenotype. Together with the null mutant, our mutant should therefore prove valuable for examining the consequences of different 
A
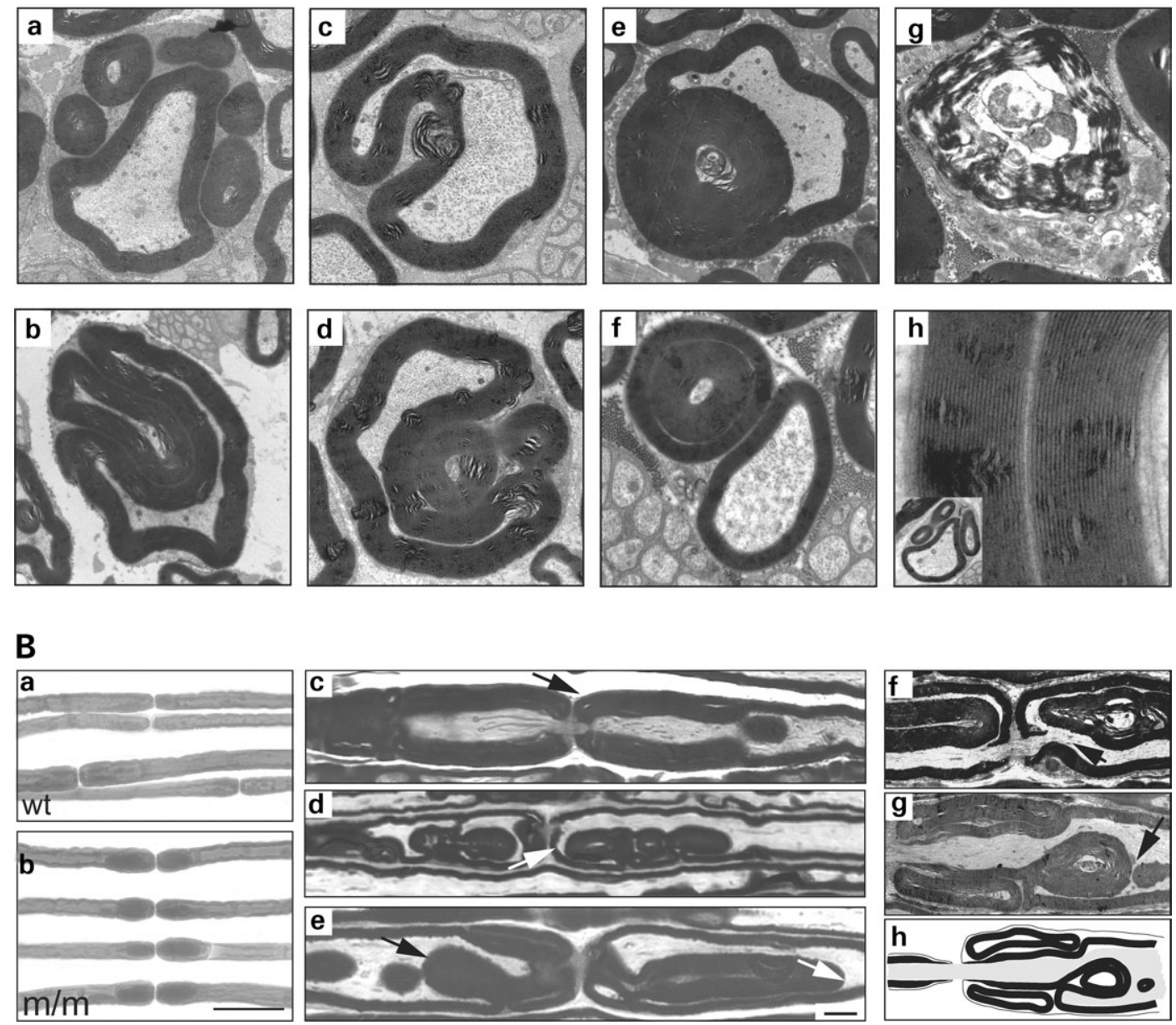

Figure 6. Focally folded myelin in Mtmr2 m/m sciatic nerve. (A) Electron microscopic analysis of sciatic nerves derived from 6-month-old Mtmr2 m/m mice. The figure shows a collection of the different types of focally folded myelin. Single or multiple abundant myelin loops aligned around the original myelinated axon are the most frequent forms of abnormal myelin (a). Redundant myelin can appear as elongated loops arranged sausage-like around the axon (b). Commalike extensions of the original myelinated fibers (c) are more frequently detectable in $M t m r 2 \mathrm{~m} / \mathrm{m}$ mice compared with wt. They can reach sizes sufficient to surround other aberrant myelin loops (d) or the original axon (data not shown). Focal abundant myelin loops can also be found inside a myelinated axon as single or multiple loops nested into each other (e). Interleaved myelin loops can also be observed (f). Rarely, degenerating axon-myelin units (g) are detectable. The number of lamella is the same in the outfoldings compared to the core myelin (h). (B) Teased fiber preparations of 9-month-old wt (a) and Mtmr2 $\mathrm{m} / \mathrm{m}$ (b) sciatic nerves revealed an increased number of nodes of Ranvier that are affected with redundant myelin. A collection of affected nodes are shown in longitudinal sections in (c-e). The additional myelin can be folded inwards (black arrow in $\mathrm{c}$ and e) or outwards (white arrow in d and e). Note that the nodes are not affected symmetrically. Aberrant myelin foldings are extensions of the original axon/myelin unit ( $\mathrm{f}$ and $\mathrm{g}$ ). A schematic drawing of the situation in ( $\mathrm{g}$ ) is shown in the right part of (h) for illustration, together with a schema for a normal node for comparison on the left. Scale bars for (a and b): $5 \mu \mathrm{m}$; for (c-e): $6 \mu \mathrm{m}$.

Mtmr2 mutations in terms of physiological disturbances and genetic background.

\section{MATERIALS AND METHODS}

\section{Generation of the Mtmr2 mutant allele}

Vector construction: a mouse genomic clone including the Mtmr 2 gene (BAC clone number 26987, Incyte) served as starting point. The short arm was generated by PCR using the BAC clone as template, with forward primer SAfo: 5'-ATA AGA ATG CGG CCG CGG CGC GCC CTG AGG GCT CCT GTG AGA C-3', with NotI and AscI restriction sites introduced for cloning and vector linearization at the $5^{\prime}$ end, and reverse primer SAba: 5'-GAT CTC TAG ATC
ACT ATT AAG GAT GAA TCC AGG A-3', with an XbaI restriction sequence at the $3^{\prime}$ end for cloning. The PCR fragment was cloned via NotI-XbaI into the IRES-lacZ-tk-neo cassette-vector backbone (37). The long arm was generated by EcoRI digestion of the BAC clone and isolation of a $6.5 \mathrm{~kb}$ fragment containing exons $13-15$ of $M t m r 2$. This EcoRI fragment was subcloned into the pGEM-T-Easy vector (Promega). Using the restriction sites XhoI and SalI of this vector, the long arm was inserted into the short arm-IRES-lacZ-tk-neo cassette backbone. The final construct was linearized, electroporated into TC-1 ES cells (129SvS6/ SvEvTac), and clones were selected with G418. Growing clones were analyzed first by PCR. Eleven positive clones were further analyzed by Southern blotting for correct and single integration. Two clones were used for blastocyst 
A
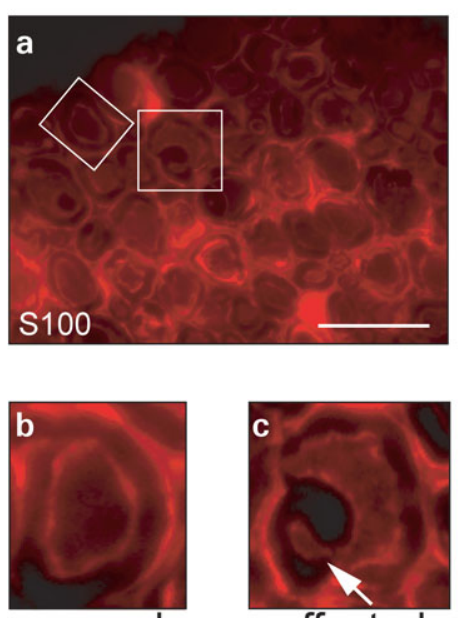

normal

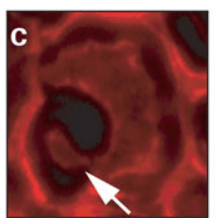

affected

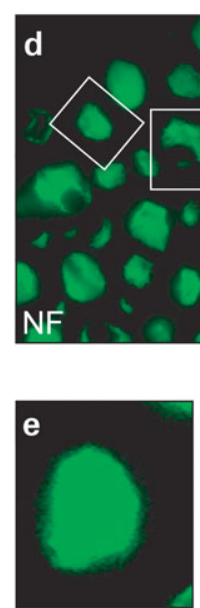

normal

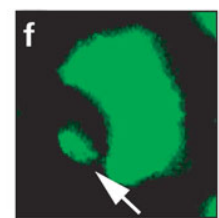

affected
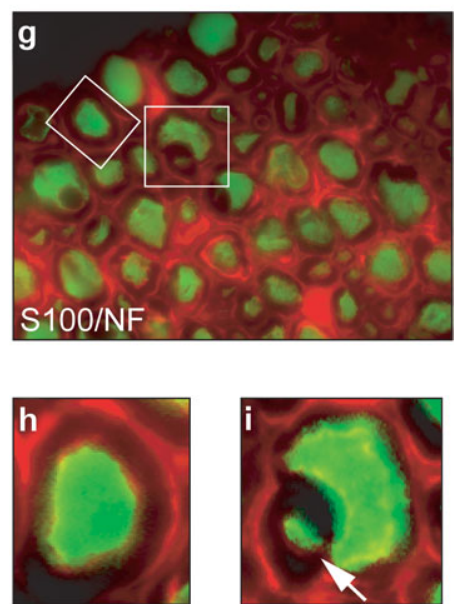

normal

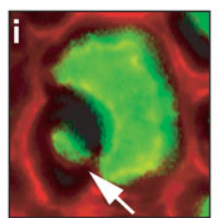

affected

B
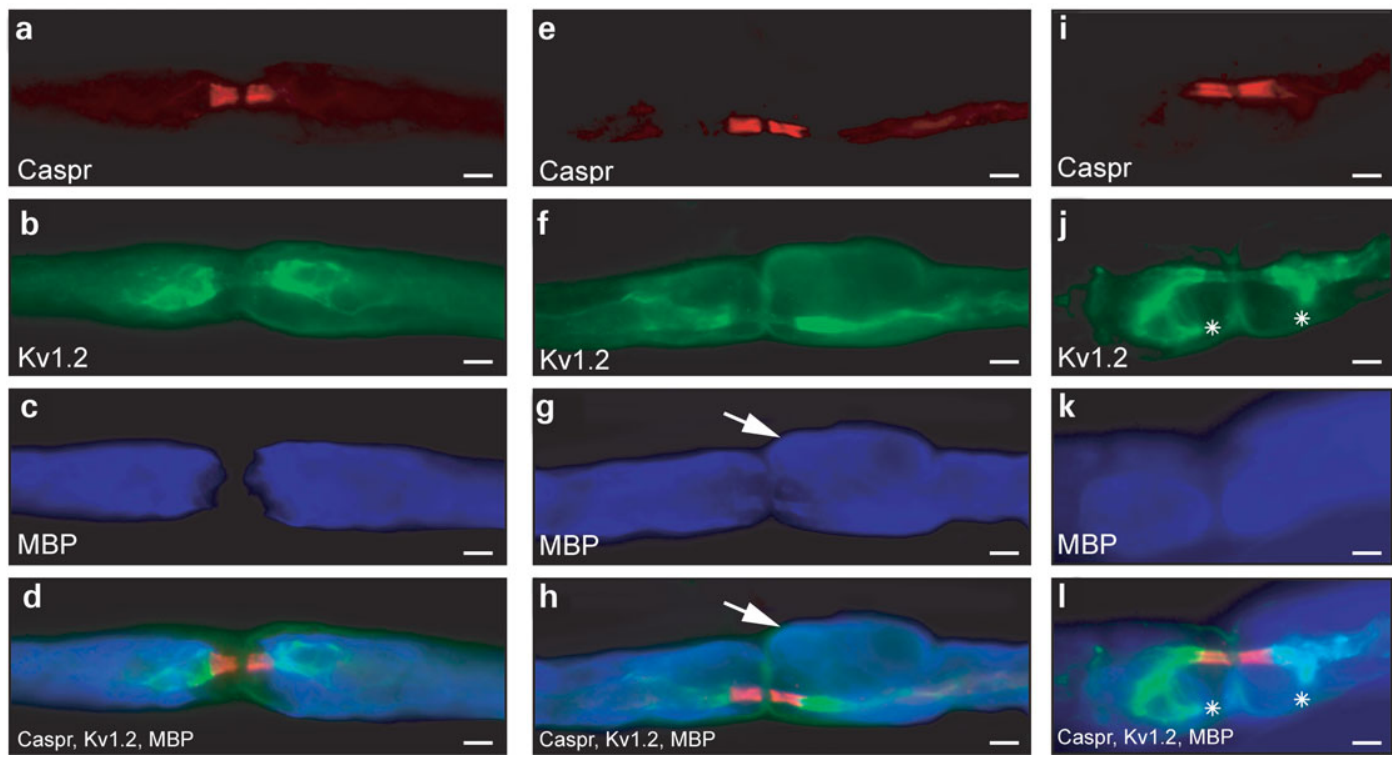

Figure 7. Immunohistochemical analysis of Mtmr $2 \mathrm{~m} / \mathrm{m}$ sciatic nerves. (A) Colocalization study on sciatic nerves of 9-month-old $\mathrm{Mtmr} 2 \mathrm{~m} / \mathrm{m}$ mice using S100 as a Schwann cell marker $(\mathrm{a}-\mathrm{c})$ and NF-L as an axonal marker $(\mathrm{d}-\mathrm{f})$. Overlays are shown in $(\mathrm{g}-\mathrm{i})$. The lower panels represent single myelinated fibers indicated in the overviews. A normal fiber is shown in (b, e and $\mathrm{h}$ ). The Schwann cell in c surrounds not only a single ring of myelin (remains black in this staining) with the corresponding axon (marked with NF-L in f), but also an additional loop (arrow in c). Remarkably, this redundant myelin surrounds an axonal, NF-L positive structure (arrow in f). Scale bar for (a, $\mathrm{d}$ and g): $20 \mu \mathrm{m}$. (B) Analysis of sciatic nerve teased fiber preparation from 9-month-old Mtmr2 $\mathrm{m} / \mathrm{m}$ mice. Localization of Caspr ( $a$, e and i), Kv1.2 (b, f and j) and MBP (c, g and k) was assessed to determine the influence of aberrant myelin foldings on the organization of the node of Ranvier. In (a-d), a node without myelin aberration shows normal localization of Caspr, Kv1.2 and MBP. A nodal region affected with an outfolding is shown in $(\mathrm{e}-\mathrm{h})$. An outfolding is present above the right side of the node (arrows) but does not change the localization of Caspr and Kv1.2. The node in (i-1) is affected by outfoldings on both sides of the node. Here, staining for the marker Kv1.2 extends into the outfolding (asterisks in $\mathrm{j}$ and $\mathrm{l}$ ). Scale bars $(\mathrm{a}-1): 1 \mu \mathrm{m}$.

injection (Institute for Laboratory Animal Science, University of Zurich) to obtain chimeric mice. These were crossed with wt $\mathrm{C} 57 \mathrm{~B} 1 / 6$ mice. Both ES cell clones resulted in mouse lines (sb65 and $\mathrm{sb59)}$. These produced offspring at the expected frequencies (line sb65: wt: $25.6 \%$; heterozygous: 48.8\%; homozygous: $25.6 \%$ with a total of 207 animals; line sb59: wt: $27 \%$; heterozygous: $48.6 \%$; homozygous: $24.3 \%$ with a total of 37 animals analyzed).

\section{Genotyping PCR}

Primers specific for the wt allele are W1: 5'-GTG TGT ATT TAC TAC TCT TTC TC-3' and W2: $5^{\prime}$-GCA GTT TGG TTA GAT ATG TGA CCT G- ${ }^{\prime}$. Primers for the detection of the mutated allele are K1: $5^{\prime}$-GCA TGC ATA CCT ATC TCT ATA TAG C-3' and K2: 5'-AAC GCA CGG GTG TTG GGT CG-3'. 


\begin{tabular}{|c|c|c|c|}
\hline & $\begin{array}{l}\text { CMAP } \\
\text { amplitude } \\
(\mathrm{mV})\end{array}$ & $\begin{array}{l}\text { F-wave } \\
\text { latency } \\
(\mathrm{ms})\end{array}$ & $\begin{array}{l}\mathrm{NCV} \\
(\mathrm{m} / \mathrm{s})\end{array}$ \\
\hline wt 8 mo & $\begin{array}{l}\text { prox. } 8.5 \pm 3.2 \\
\text { dist. } 11.8 \pm 3.3\end{array}$ & $4.2 \pm 0.5$ & $47.1 \pm 5.8$ \\
\hline $\mathrm{m} / \mathrm{m} 8 \mathrm{mo}$ & $\begin{array}{lc}\text { prox } & 6.3 \pm 2.1 \\
\text { dist. } & 10 \pm 3.8\end{array}$ & $4.6 \pm 0.3$ & $51.9 \pm 4.0$ \\
\hline wt $16 \mathrm{mo}$ & $\begin{array}{ll}\text { prox. } & 5.7 \pm 2.5 \\
\text { dist. } & 6.3 \pm 3.2\end{array}$ & $4.3 \pm 0.4$ & $57.7 \pm 9.7$ \\
\hline $\mathrm{m} / \mathrm{m} 16 \mathrm{mo}$ & $\begin{array}{ll}\text { prox } & 5.2 \pm 2.1 \\
\text { dist. } & 7.1 \pm 2.9\end{array}$ & $4.5 \pm 0.5$ & $57.2 \pm 10.7$ \\
\hline
\end{tabular}

Figure 8. Electrophysiological analysis. NCV, CMAPs and F-wave latencies are not significantly altered in the sciatic nerves of $M t m r 2 \mathrm{~m} / \mathrm{m}$ mice up to the age of 16 months.

\section{Histological analysis}

For semithin sections and electron microscopy, mice of different ages were anesthetized with pentobarbital (Eutha77; Essex) and perfused with $0.1 \mathrm{M}$ phosphate buffer $\mathrm{pH} 7.2$ containing $4 \%$ PFA and $3 \%$ glutaraldehyde. Samples were post-fixed overnight in the same fixative at $4^{\circ} \mathrm{C}$. Samples were then washed in phosphate buffer, treated with $2 \%$ $\mathrm{OsO}_{4}$ overnight at $4{ }^{\circ} \mathrm{C}$, dehydrated in an ascending acetone series, infiltrated with Spurr and incubated at $60^{\circ} \mathrm{C}$ for polymerization. Semithin sections $(0.5 \mu \mathrm{m})$ were stained with $0.1 \%$ Toluidine blue. Electron microscopic pictures were taken digitally with a Philips Fei Morgani using ANALYSIS software (Soft Imaging System, Münster, Germany). Pictures were processed in Photoshop.

Teased $\mathrm{OsO}_{4}$-fixed fibers were obtained by separating single fibers of sciatic nerves after the ascending acetone series and infiltration with Spurr.

For immunohistochemistry, isolated nerves were frozen in OCT and cut to $7 \mu \mathrm{m}$ sections, which were then fixed with $4 \%$ PFA. For teased fibers, nerves were fixed in 4\% PFA for $30 \mathrm{~min}$ at room temperature, and fibers then separated in PBS. Teased fibers were permeabilized in ice-cold acetone prior to the blocking step for $10 \mathrm{~min}$. Both sections and teased fibers were blocked with $1 \%$ BSA $/ 0.3 \%$ Triton $\mathrm{X}-100 / 10 \%$ goat serum. Primary antibodies were diluted in blocking solution and incubated overnight at $4^{\circ} \mathrm{C}$ [rabbit polyclonal anti-S100 1:300 (DAKO), mouse monoclonal anti-NFlight chain 1:40 (Sigma), rabbit polyclonal anti-Caspr 1:500 (a kind gift from Drs L. Pedraza and D. Colman, Montreal), mouse monoclonal anti-Kv1.2 1:50 (Upstate Biotechnology), rat monoclonal anti-MBP 1:50 (Serotec)]. Secondary antibodies were incubated in blocking solution for $1 \mathrm{~h}$ at room temperature [goat anti-rabbit $\mathrm{Cy} 3$ 1:300; goat anti-mouse Cy2 1:200; goat anti-rat Cy5 1:300 (Jackson Immuno Research Laboratories)]. Specimens were mounted in Citiflour (Canterbury, UK) and analyzed on a Zeiss Axioplan microscope. Pictures were taken with a Zeiss Axiocam and processed in Photoshop.

\section{G-ratios}

Three animals per genotype were analyzed at each age ( 3 weeks and 6 months). Sciatic nerves were processed to $0.5 \mu \mathrm{m}$ semithin sections and stained with Toluidine blue. The axon caliber and the myelin sheath thickness were determined using the ANALYSIS software. We evaluated only fibers with normally compacted myelin (extending over the entire thickness of the sheath) and excluded fibers with prominent myelin foldings. Myelin thickness of sciatic nerve fibers was plotted as a function of the corresponding axon diameter. Statistical analysis for this and the tests below was performed using Student's $t$-test.

\section{Rotarod test}

Mice were placed on a Rotarod apparatus (TSE Systems, Germany), and the rod was accelerated from 4 to 40 r.p.m. Time spent on the rotating rod was measured. The trial was stopped after $10 \mathrm{~min}$. A mouse not running but spinning on the rotating rod two or more times was considered to be exhausted, and the trial was stopped.

\section{Grip strength analysis}

Mutant and control mice were tested five consecutive times. Each time mice were allowed to grab a movable metal grid. They are then lifted by the tail and slightly pulled back for $1 \mathrm{~min}$. The peak weight that the mouse could hold was recorded (in grams). The maximum value was then divided by the body weight of the mouse.

\section{Nerve conduction studies}

Motor nerve conduction of sciatic nerves in anesthetized 8- and 16-month-old wt and mutant mice ( $n=6$ in each group) was investigated by a robust technique as described previously (5). After supramaximal stimulation of the tibial nerve at the ankle and stimulation of the sciatic notch, the CMAPs were recorded with steel needle electrodes in the foot muscles.

\section{ACKNOWLEDGEMENTS}

We thank Joke Nowitzki for excellent technical assistance and Drs L. Pedraza and D. Colman for reagents. This work has been supported by the Deutsche Forschungsgemeinschaft (DFG Y048/3-1), the Swiss National Science Foundation and the NCCR Neural Plasticity and Repair.

Conflict of Interest statement. The authors declare that they have no conflict of interest regarding this manuscript.

\section{REFERENCES}

1. Suter, U. and Snipes, G.J. (1995) Biology and genetics of hereditary motor and sensory neuropathies. Ann. Rev. Neurosci., 18, 45-75.

2. Suter, U. and Scherer, S.S. (2003) Disease mechanisms in inherited neuropathies. Nat. Rev. Neurosci., 4, 714-726.

3. Martini, R. (2000) Animal models for inherited peripheral neuropathies: chances to find treatment strategies? J. Neurosci. Res., 61, 244-250. 
4. Lewis, R.A., Sumner, A.J. and Shy, M.E. (2000) Electrophysiological features of inherited demyelinating neuropathies: a reappraisal in the era of molecular diagnosis. Muscle Nerve, 23, 1472-1487.

5. Zielasek, J., Martini, R. and Toyka, K.V. (1996) Functional abnormalities in P0-deficient mice resemble human hereditary neuropathies linked to P0 gene mutations. Muscle Nerve, 19, 946-952.

6. Bolino, A., Muglia, M., Conforti, F.L., LeGuern, E., Salih, M.A., Georgiou, D.M., Christodoulou, K., Hausmanowa-Petrusewicz, I., Mandich, P., Schenone, A. et al. (2000) Charcot-Marie-Tooth type 4B is caused by mutations in the gene encoding myotubularin-related protein- 2 . Nat. Genet., 25, 17-19.

7. Houlden, H., King, R.H., Wood, N.W., Thomas, P.K. and Reilly, M.M. (2001) Mutations in the $5^{\prime}$ region of the myotubularin-related protein 2 (MTMR2) gene in autosomal recessive hereditary neuropathy with focally folded myelin. Brain, 124, 907-915.

8. Nelis, E., Erdem, S., Tan, E., Lofgren, A., Ceuterick, C., De Jonghe, P., Van Broeckhoven, C., Timmerman, V. and Topaloglu, H. (2002) A novel homozygous missense mutation in the myotubularin-related protein 2 gene associated with recessive Charcot-Marie-Tooth disease with irregularly folded myelin sheaths. Neuromuscul. Disord., 12, 869-873.

9. Kochanski, A., Drac, H., Jedrzejowska, H. and Hausmanowa-Petrusewicz, I. (2003) Focally folded myelin in CharcotMarie-Tooth type 1B disease is associated with Asn131Lys mutation in myelin protein zero gene: short report. Eur. J. Neurol., 10, 547-549.

10. Fabrizi, G.M., Taioli, F., Cavallaro, T., Rigatelli, F., Simonati, A., Mariani, G., Perrone, P. and Rizzuto, N. (2000) Focally folded myelin in Charcot-Marie-Tooth neuropathy type 1B with Ser49Leu in the myelin protein zero. Acta Neuropathol. (Berl.), 100, 299-304.

11. Berger, P., Bonneick, S., Willi, S., Wymann, M. and Suter, U. (2002) Loss of phosphatase activity in myotubularin-related protein 2 is associated with Charcot-Marie-Tooth disease type 4B1. Hum. Mol. Genet., 11, $1569-1579$

12. Bolino, A., Marigo, V., Ferrera, F., Loader, J., Romio, L., Leoni, A., Di Duca, M., Cinti, R., Cecchi, C., Feltri, M.L. et al. (2002) Molecular characterization and expression analysis of $\mathrm{Mtmr}$, mouse homologue of MTMR2, the myotubularin-related 2 gene, mutated in CMT4B. Gene, 283, 17-26.

13. Bolino, A., Bolis, A., Previtali, S.C., Dina, G., Bussini, S., Dati, G., Amadio, S., Del Carro, U., Mruk, D.D., Feltri, M.L. et al. (2004) Disruption of Mtmr2 produces CMT4B1-like neuropathy with myelin outfolding and impaired spermatogenesis. J. Cell Biol., 167, 711-721.

14. Laporte, J., Bedez, F., Bolino, A. and Mandel, J.L. (2003) Myotubularins, a large disease-associated family of cooperating catalytically active and inactive phosphoinositides phosphatases. Hum. Mol. Genet., 12 (Spec no. 2), R285-R292.

15. Berger, P., Schaffitzel, C., Berger, I., Ban, N. and Suter, U. (2003) Membrane association of myotubularin-related protein 2 is mediated by a pleckstrin homology-GRAM domain and a coiled-coil dimerization module. Proc. Natl Acad. Sci. USA, 100, 12177-12182.

16. Previtali, S.C., Zerega, B., Sherman, D.L., Brophy, P.J., Dina, G., King, R.H., Salih, M.M., Feltri, L., Quattrini, A., Ravazzolo, R. et al. (2003) Myotubularin-related 2 protein phosphatase and neurofilament light chain protein, both mutated in CMT neuropathies, interact in peripheral nerve. Hum. Mol. Genet., 12, 1713-1723.

17. Azzedine, H., Bolino, A., Taieb, T., Birouk, N., Di Duca, M., Bouhouche, A., Benamou, S., Mrabet, A., Hammadouche, T., Chkili, T. et al. (2003) Mutations in MTMR13, a new pseudophosphatase homologue of MTMR2 and Sbf1, in two families with an autosomal recessive demyelinating form of Charcot-Marie-Tooth disease associated with early-onset glaucoma. Am. J. Hum. Genet., 72, $1141-1153$

18. Senderek, J., Bergmann, C., Weber, S., Ketelsen, U.P., Schorle, H., Rudnik-Schoneborn, S., Buttner, R., Buchheim, E. and Zerres, K. (2003) Mutation of the $S B F 2$ gene, encoding a novel member of the myotubularin family, in Charcot-Marie-Tooth neuropathy type 4B2/11p15. Hum. Mol. Genet., 12, 349-356.

19. Nandurkar, H.H., Layton, M., Laporte, J., Selan, C., Corcoran, L., Caldwell, K.K., Mochizuki, Y., Majerus, P.W. and Mitchell, C.A. (2003) Identification of myotubularin as the lipid phosphatase catalytic subunit associated with the 3-phosphatase adapter protein, 3-PAP. Proc. Natl Acad. Sci. USA, 100, 8660-8665.

20. Mochizuki, Y. and Majerus, P.W. (2003) Characterization of myotubularin-related protein 7 and its binding partner, myotubularinrelated protein 9. Proc. Natl Acad. Sci. USA, 100, 9768-9773.

21. Kim, S.A., Vacratsis, P.O., Firestein, R., Cleary, M.L. and Dixon, J.E. (2003) Regulation of myotubularin-related (MTMR)2 phosphatidylinositol phosphatase by MTMR5, a catalytically inactive phosphatase. Proc. Natl Acad. Sci. USA, 100, 4492-4497.

22. Robinson, F.L. and Dixon, J.E. (2005) The phosphoinositide 3-phosphatase MTMR2 associates with MTMR13, a novel membrane-associated pseudophosphatase also mutated in type 4B Charcot-Marie-Tooth disease. J. Biol. Chem., 280, 31699-31707.

23. Walker, D.M., Urbe, S., Dove, S.K., Tenza, D., Raposo, G. and Clague, M.J. (2001) Characterization of MTMR3, an inositol lipid 3-phosphatase with novel substrate specificity. Curr. Biol., 11, $1600-1605$

24. Schaletzky, J., Dove, S.K., Short, B., Lorenzo, O., Clague, M.J. and Barr, F.A. (2003) Phosphatidylinositol-5-phosphate activation and conserved substrate specificity of the myotubularin phosphatidylinositol 3-phosphatases. Curr. Biol., 13, 504-509.

25. Sereda, M.W., Meyer zu Horste, G., Suter, U., Uzma, N. and Nave, K.A. (2003) Therapeutic administration of progesterone antagonist in a model of Charcot-Marie-Tooth disease (CMT-1A). Nat. Med., 9, 1533-1537.

26. Passage, E., Norreel, J.C., Noack-Fraissignes, P., Sanguedolce, V., Pizant, J., Thirion, X., Robaglia-Schlupp, A., Pellissier, J.F. and Fontes, M. (2004) Ascorbic acid treatment corrects the phenotype of a mouse model of Charcot-Marie-Tooth disease. Nat. Med., 10, 396-401.

27. Sahenk, Z., Nagaraja, H.N., McCracken, B.S., King, W.M., Freimer, M.L., Cedarbaum, J.M. and Mendell, J.R. (2005) NT-3 promotes nerve regeneration and sensory improvement in CMT1A mouse models and in patients. Neurology, 65, 681-689.

28. Martini, R. and Toyka, K.V. (2004) Immune-mediated components of hereditary demyelinating neuropathies: lessons from animal models and patients. Lancet Neurol., 3, 457-465.

29. Benstead, T.J. and Grant, I.A. (2001) Progress in clinical neurosciences: Charcot-Marie-Tooth disease and related inherited peripheral neuropathies. Can. J. Neurol. Sci., 28, 199-214.

30. Kim, S.A., Taylor, G.S., Torgersen, K.M. and Dixon, J.E. (2002) Myotubularin and MTMR2, phosphatidylinositol 3-phosphatases mutated in myotubular myopathy and type 4B Charcot-Marie-Tooth disease. J. Biol. Chem., 277, 4526-4531.

31. Parrish, W.R., Stefan, C.J. and Emr, S.D. (2004) Essential role for the myotubularin-related phosphatase Ymrlp and the synaptojanin-like phosphatases Sj12p and Sj13p in regulation of phosphatidylinositol 3-phosphate in yeast. Mol. Biol. Cell, 15, 3567-3579.

32. Xue, Y., Fares, H., Grant, B., Li, Z., Rose, A.M., Clark, S.G. and Skolnik, E.Y. (2003) Genetic analysis of the myotubularin family of phosphatases in Caenorhabditis elegans. J. Biol. Chem., 278, $34380-34386$.

33. Dang, H., Li, Z., Skolnik, E.Y. and Fares, H. (2004) Disease-related myotubularins function in endocytic traffic in Caenorhabditis elegans. Mol. Biol. Cell, 15, 189-196.

34. Tsujita, K., Itoh, T., Ijuin, T., Yamamoto, A., Shisheva, A., Laporte, J. and Takenawa, T. (2004) Myotubularin regulates the function of the late endosome through the GRAM domain-phosphatidylinositol 3,5-bisphosphate interaction. J. Biol. Chem., 279, 13817-13824.

35. Lee, O.K., Frese, K.K., James, J.S., Chadda, D., Chen, Z.H., Javier, R.T. and Cho, K.O. (2003) Discs-large and strabismus are functionally linked to plasma membrane formation. Nat. Cell Biol., 5, 987-993.

36. Firestein, R., Nagy, P.L., Daly, M., Huie, P., Conti, M. and Cleary, M.L. (2002) Male infertility, impaired spermatogenesis, and azoospermia in mice deficient for the pseudophosphatase Sbf1. J. Clin. Invest., 109, $1165-1172$.

37. Schaeren-Wiemers, N., Bonnet, A., Erb, M., Erne, B., Bartsch, U., Kern, F., Mantei, N., Sherman, D. and Suter, U. (2004) The raft-associated protein MAL is required for maintenance of proper axon-glia interactions in the central nervous system. J. Cell Biol., 166, 731-742. 